\title{
ENZIMAS MICROBIANA: POTENCIAL BIOTECNOLÓGICO E EFEITOS MODULATÓRIOS EM RUMINANTES
}

Flávia Oliveira Abrão; Cláudio Eduardo Silva Freitas²; Eduardo Robson Duarte³ Moisés Sena Pessoa ${ }^{4}$; Luis Henrique Curcino Batista ${ }^{5}$; Thiago Dias Silva ${ }^{5}$; Eliane Vieira Rosa ${ }^{1}$; Fillipe Hebert de Oliveira $^{7}$

${ }^{1}$ Instituto Federal Goiano, Campus Ceres, GO, Brasil. ${ }^{2}$ Universidade Estadual do Sudoeste da Bahia, Pós-Graduação em Zootecnia, BA. ${ }^{3}$ Universidade Federal de Minas Gerais - UFMG, Instituto de Ciências Agrárias, campus Montes Claros, MG. ${ }^{4}$ Universidade Federal de Goiás - UFG, Pós-Graduação em Zootecnia, GO. ${ }^{5}$ Instituto Federal Goiano, Curso de Zootecnia, Campus Ceres, GO. ${ }^{7}$ Universidade de Brasília - UNB, Brasília, DF. E-mail: flavia.abrao@ifgoiano.edu.br.

\section{RESUMO}

Objetivou-se com a presente revisão levantar as principais espécies microbianas produtoras de enzimas envolvidas na degradação de compostos como a celulose, hemicelulose e lignina. A maioria dos microrganismos isolados do solo, de resíduos e material de compostagem são capazes de produzir um amplo espectro de enzimas degradantes da parede celular vegetal. Essa atividade enzimática é essencial para a fermentação de carboidratos no ambiente ruminal, uma vez que os ruminantes não são capazes de degradar esses polímeros, estabelecendo assim uma relação de simbiose entre esses animais e a microbiota autóctone do trato gastrointestinal. Contudo, não somente na nutrição animal as enzimas microbianas tem recebido atenção, e cada vez mais estas tem sido exploradas pelo setor industrial quanto as possibilidades biotecnologicas, como a produção de etanol. Observa-se uma diversidade microbiana no rúmen, e variações na produção de enzimas. Mais estudos fazem-se necessários para elucidar os mecanismos modulatórios de enzimas no rúmen de animais de produção, a fim de se obter aumento nos índices zootécnicos e uma maior digestibilidade da fibra.

Palavras-Chave: Celulose, hemicelulose, lignina, rúmen.

\section{MICROBIAL ENZYMES: BIOTECHNOLOGICAL POTENCIAL AND MODULATORY EFFECTS IN RUMINANTS}

\begin{abstract}
The objective of this review was to survey the main microbial species producing enzymes involved in the degradation of compounds such as cellulose, hemicellulose and lignin. Most of the microorganisms isolated from soil, waste and compost material are capable of producing a broad spectrum of degrading enzymes from the plant cell wall. This enzymatic activity is essential for the fermentation of carbohydrates in the ruminal environment, since ruminants are not able to degrade these polymers, thus establishing a symbiosis relationship between these animals and the autochthonous microbiota of the gastrointestinal tract. However, microbial enzymes have not only been receiving attention in animal nutrition, more and more these have been explored by the industry as biotechnological possibilities, such as the production of ethanol. It is observed a microbial diversity in the rumen, and variations in the production of enzymes. Further studies are needed to elucidate the mechanisms of enzyme modulation in the rumen of production animals, in order to obtain an increase in zootechnical indexes and a higher digestibility of the fiber.
\end{abstract}

Keywords: Cellulose, hemicellulose, lignin, rumen. 


\section{INTRODUÇÃO}

Lignocelulose é um dos abundantes complexos naturais de carbono orgânico encontrado na forma de biomassa vegetal, que consiste principalmente em três componentes vegetais: hemicelulolse, celulose e lignina. A celulose é um homopolissacarídeo $(\beta-1,4)$ ligado a resíduos de D-glucose, com xilana como seu principal componente, podendo apresentar variações de acordo com a espécie da planta (BADHAN et al., 2007; WANG et al., 2014).

A estrutura variável e organização da hemicelulose exigem a ação sinérgica de várias enzimas para a sua completa degradação. Este processo é muito lento devido à estrutura rígida insolúvel da parede celular da planta e à disponibilidade limitada de microrganismos celulolíticos e hemicelulolítcos eficientes. O polímero de hemicelulose possui alto peso molecular, pode ser insolúvel ou associado a celulose e a lignina. Como a hidrólise dos polissacarídeos ocorre fora da célula, os microrganismos têm de assegurar que pelo menos parte dos açúcares solúveis resultantes do processo de digestão estarão disponíveis para eles (SHALLOM; SHOHAM, 2003).

A degradação da celulose e hemicelulose é realizada por microrganismos que podem ser encontrados naturalmente no ambiente ou no trato digestório dos animais (SHALLOM; SHOHAM, 2003; GUTIÉRREZ-ROJAS et al., 2015). A maioria dos microrganismos isolados do solo, de resíduos e material de compostagem são capazes de produzir um amplo espectro de enzimas degradantes da parede celular vegetal (BADHAN et al., 2007).

A hidrólise da celulose é realizada por componentes da celulase. Além disso, diferentes enzimas, como por exemplo, arabinofuranosidase, glucouronidase, acetil esterases e acetil xilana esterases, também são necessárias para uma hidrólise eficaz da fração hemicelulósica. Essas enzimas têm aplicações em rações animais e nas industrias têxtil e alimentícias, bem como nas de papel e celulose. As possibilidades de aplicações também incluem bioconversão de materiais lignocelulósicos e agro-resíduos de produtos de fermentação, clarificação de sucos e melhoria na consistência de cervejas (BADHAN et al., 2007).

Poucos são os trabalhos que compilam informações a cerca das enzimas microbianas sobre a fibra ingrediente da dieta de animais em sistema extensivo de pastejo, bem como sobre as possibilidades de utilização na modulação dos parâmetros fermentativos ruminais. Dessa forma, evidencia-se a necessidade de explorar o potencial papel de enzimas microbianas na área biotecnológica e, mais especificamente na nutrição animal (SUBRAMANIYAN; PREMA, 2002).

\section{REVISÃO DE LITERATURA}

\subsection{Fungos aeróbios e/ou anaeróbios facultativos celulolíticos, hemicelulolíticos e lignolíticos}

Atualmente, sabe-se que apesar da abundante disponibilidade de celulose, apenas uma pequena porcentagem de microrganismos é capaz de degrada-la completamente. Um dos grupos mais importantes corresponde ao dos fungos filamentosos, dentre eles: T. reesei, A. niger, A. nidulans e apontado mais recentemente Neurospora crassa (GUTIÉRREZ-ROJAS et al., 2015).

Fungos filamentosos possuem a capacidade de produzir um complexo de enzimas como a celulase e a xilanase. As celulases microbianas são mundialmente estudadas por vários pesquisadores com o objetivo de aumentar a liberação de glicose dos substratos, maximizando a fermentação e produção de etanol (DIJKERMAN et al., 1997).

Genes que codificam as enzimas celulolíticas e xilanolíticas foram isoladas de fungos dos gêneros Aspergillus e Trichoderma (MARUI et al., 2002). Novas linhagens de Trichoderma reesei têm sido desenvolvidas e vem como uma ferramenta útil para melhorar a atividade da celulase ou a produção da mesma (ZHANG et al., 2010).

Brewer e Taylor (1969) estudaram a população de fungos isolados aerobicamente do fluido ruminal de ovelhas criadas sob regime extensivo na Inglaterra. Estes pesquisadores observaram a presença de estruturas fúngicas típicas de Aspergillus fumigatus e Sporomia spp. no fluido ruminal 
desses animais. Em estudo realizado por Abedin et al. (2013), essa espécie do gênero Aspergillus apresentou potencial máximo para degradação da lignina e atividade da lacase.

Os principais sinais indutivos para degradação da parede celular vegetal por fungos filamentosos são derivados de celulose e xilana. Marui et al. (2002) isolaram um ativador importante do gene da xilanase e avaliaram sua expressão em Aspergillus oryzae. Sabe-se que esse ativador é capaz de mediar a indução de celulose por expressão dos genes xilanolíticos, bem como a de genes celulolíticos (MAURI et al., 2002).

A produção extracelular de celulases por Aspergillus terreus M11 em materiais lignocelulósicos foi estudado. Os resultados mostraram que a atividade da celulase atingiu alto nível a $45 \circ \mathrm{C} \mathrm{e} \mathrm{pH} 3$. Endoglucanase e b-glucosidase apresentaram atividade máxima em pH 2 e pH 3 , respectivamente, e ambas mostraram notável estabilidade na faixa de $\mathrm{pH} 2-5$. As preparações de enzimas a partir desta cepa foram usadas para hidrolisar Avicel (celulose microcristalina) e os maiores índices de hidrólise foram obtidos até 63\% em Avicel (5\%) por 72 horas. Tradicionalmente, a celulose é hidrolisada em meios ácidos a altas temperaturas para produção de bioetanol. (GAO et al., 2008). Mostafa et al. (2016) corroboram com o fato de que A. Terreus tem se mostrado um bom produtor de celulase, contudo neste trabalho, foi demonstrado atividade enzimática ótima em temperatura variando de 55 a $60^{\circ} \mathrm{C}$, e pH 5,5 a 6,0.

Acremonium cellulolyticus é um fungo produtor de celulase e tem sido explorado pela indústria de enzimas. Trabalhos mostram que a adição de lactose aumenta significativamente a produção de várias proteínas com atividade celulase por A. Cellulolyticus. Sabe-se ainda que celulases de Trichoderma e Aspergillus spp. têm sido investigadas em detalhes há muitas décadas. No entanto, pouco se sabe sobre celulases do Acremonium cellulolyticus (FANG et al., 2008).

Outro fungo descrito na literatura científica como destaque na degradação da celulose é o Sporotrichum thermophile. Este pode degradar alguns tipos de material celulósico mais rápido do que Trichoderma reesei (GAIKWAD; MAHESHWARI, 1994).

Neurospora crassa e Fusarium oxysporum são consideradas raras espécies de fungos que apresentam simultaneamente alta produção de celulase e de etanol celulósico. Ambas as espécies foram recentemente aprovadas para utilização em experimentos com conversão direta de lignoceluloses a etanol (LIN et al., 2010).

Em estudo recente, os fungos Tuber maculatum e Tuber aestivum foram avaliados quanto a capacidade de produção de enzimas. Nadim et al. (2015) mostraram que a primeira espécie citada produz sete tipos de enzimas (amilase, xilanase, lacase, lipase, peroxidase, cellulase and catalase), já $T$. Aestivum só foi capaz de produz amilase, peroxidase e catalase; evidenciando a variação na produção de enzimas entre espécies do mesmo gênero fúngico.

Pleurotus ostreatus e $P$. sajor-caju foram testados quanto a capacidade de produzir várias enzimas celulolíticas e ligninolíticas como a lacase, lignina peroxidase, xilanase, endo-1,4- $\beta$ - Dglucanase e exo-1,4- $\beta$-D-glucanase em resíduos agrícolas de banana (biomassa de folhas e pseudocaules). Os padrões de produção dessas enzimas extracelulares foram estudadas durante o crescimento dos microrganismos por um período de 40 dias. Ambos os microrganismos apresentaram níveis semelhantes de atividades enzimáticas. Níveis muito baixos de atividade das enzimas celulolíticas foram observados em relação as enzimas degradantes de lignina (REDDY et al., 2003).

Uma revisão realizada há mais de dez anos já apresentava características de produção de xilanase para diferentes grupos de microrganismos. Alguns dos microrganismos descritos como produtores dessa enzima foram: Aspergillus niger, Aspergillus nidulans, Fusarium oxysporum, Geotrichum candidum, Paecilomyces varioti, Penicillium purpurogenum, Trichoderma reesei, Cephalosporium sp., Trichoderma harzianum (BEG et al., 2001). 


\subsection{Fungos anaeróbios estritos do rúmen celulolíticos e lignolíticos}

Trabalhos relataram a capacidade de degradação da celulose por microrganismos ruminais. Sabe-se que as enzimas dos fungos Piromyces sp. e Neocallimastix patriciarum são capazes de converter efetivamente até $2 \%(\mathrm{w} / \mathrm{v}$ ) de celulose microcristalina (Avicel) para glicose, indicando assim a presença de todas as atividades catalíticas (endoglucanases, exoglucanases e Pglicosidases) necessárias. As enzimas de fungos anaeróbios são muito estáveis a $40^{\circ} \mathrm{C}$. Após uma semana de incubação, a essa temperatura, na ausência de substrato, aproximadamente $50 \%$ da atividade em Avicel é mantida. Vários materiais naturais lignocelulósicos foram moídos e testados como substratos. Uma correlação negativa foi encontrada entre a quantidade de lignina no substrato e da liberação de açúcares solúveis (DIJKERMAN et al., 1997).

Com o objetivo de avaliar a resistência mecânica de palha de trigo, após incubação em culturas de microrganismos ruminais celulolíticos, Fonty et al. (1999) observaram que Neocallimastix frontalis reduziu consideravelmente a resistência à fração da palha, enquanto bactérias ruminais como Ruminococcus flavefaciens e Fibrobacter succinogenes não conseguiram promover essa redução.

Srinivasan et al. (2001) determinaram a produção de enzimas celulolíticas e xilanolíticas de Neocallimastix frontalis em meios de cultura contendo diferentes concentrações de celulose. A produção de enzimas celulolíticas e os produtos da fermentação não diferiram quando esse fungo foi cultivado em meios contendo $8 \mathrm{~g}$ e $4 \mathrm{~g}$ de celulose, respectivamente.

Pesquisas revelam ainda que Piromyces e Neocallimastix patriciarum possuem níveis significativamente mais elevados de atividade fibrolítica que Orpinomyces joyonii e Neocallimastix frontalis (YANKE et al., 1996). Ademais, o Neocallimastix spp. é um dos principais fungos anaeróbios no rúmen capaz de digerir eficientemente a biomassa celulósica (WANG et al., 2011).

\subsection{Bactérias com potencial celulolítico e hemicelulolítico e xilanolítico}

Bactérias com produção característica de celulase têm sido estudas por muitos pesquisadores. Estes microrganismos são encontrados nos mais diversos ambientes. Porém, o elevado custo de produção dessas enzimas tem dificultado a sua aplicação na indústria de bioconversão (NARASIMHA et al., 2006).

Para as bactérias, observa-se uma diferença na estrátegia celulolítica entre os grupos aeróbios e anaeróbios. As bactérias aeróbias celulolíticas do gênero Acidothermus, Bacillus, Cellulomonas, Streptomyces, Pseudomonas e Micromonospora degradam a celulose principalmente por sistemas de celulases, estas enzimas podem degradar a forma cristalina deste polissacarídeo. As bactérias anaeróbias celulolíticas do gênero Anaerocellum, Clostridium, Fibrobacter, Butyrivibrio e Ruminococcus possuem complexos "celulossomas" localizados em sua superficie que aderem ao substrato, sendo esta adesão especifica para a celulose. O mecanismo de degradação da celulose por bactérias celuloliticas aeróbias é semelhante ao dos fungos aeróbios, porém estas utilizam um sistema diferente (COUGHLAN; LUNGDAHL, 1988; KARMAKAR, 2010).

Amostras obtidas de um aterro sanitário na cidade de Laval na França revelaram uma alta diversidade fenotípica de bactérias celulolíticas, que foram distribuídas em 21 grupos, com base na análise fenotípica e sequenciamento do DNAr 16s de cinco estirpes representativas; os grupos celulolíticos predominantes foram atribuídos à família de Bacillaceae e aos gêneros Cellulomonas, Microbacterium e Lactobacillus (POURCHER et al., 2001).

A bactéria Gram-positiva Streptomyces reticuli que tem sido utilizada há anos na indústria farmacêutica, pois produz uma grande variedade de antibióticos quimicamente diferentes, quando cultivada com celulose cristalina, sendo esta a única fonte de carbono, produziu micelos e dregadou a celulose cristalina a celobiose (WALTER, 1995). 
Estirpes de Clostridium thermocellum ACTP 27405, 10682 e NCZB LQRI têm sido examinadas para sacarificação de diferentes formas de celulose e avaliadas quanto a capacidade de produção de celulase. A cepa ATCC 27405 foi a mais efetiva no metabolismo de celulose em papel filtro e celulose CFIl (o substrato mais resistente) (HALLIWEL, 1995).

A degradação da celulose e o metabolismo no rúmen podem ser negativamente afetados pela presença de açúcares solúveis. A bactéria ruminal Ruminococcus albus foi incubada com uma combinação de celobiose e glicose, o microrganismo utilizou preferencialmente o dissacarídeo. Essa preferência pode estar relacionada à repressão dos sistemas de captação de glicose nas células de celobiose crescidas (THURSTON, 1993).

Estudos demonstraram que apenas Fibrobacter succionogenes, Ruminococcus flavefaciens e Ruminococcus albus são constantemente encontradas no rúmen e realizam intensa solubilização de celulose e hemicelulose. Uma comprovação dessa atividade pode ser o fato de que $F$. succionogenes tem sido encontrada preferencialmente associada a fibras de celulose (FLINT, 1994).

A bactéria Bacillus pumilus produz enzimas xilanolíticas que apresentam efeito de branqueamento na polpa kraft da indústria de papel. Estudos in vitro com a bactéria Bacillus pumilus demonstrou a capacidade de produção de xilanases quando há aumento dos níveis de glicose, a atividade xilanásica cresceu com uma concentração de glicose de $0,6 \%$ o que indica que uma baixa concentração de glicose é necessária para ativar a atividade enzimática (AMANI, 2007).

Outras espécies microbianas que têm sido associadas a degradação de xilana no rúmen são: Butyrivibrio fibrisolvens, Bacteroides graminisolvens, Prevotella ruminicola, Pseudobutyrivibrio xylanivorans, Eubacterium uniforme (BABA et al., 2016).

\subsection{Enzimas microbianas e sua relação com o metabolismo ruminal}

Sabe-se que diversos microrganismos têm sido estudados e avaliados na dieta de diferentes espécies animais como possíveis probióticos ou aditivos microbianos. Para que diferentes microrganismos possam ser utilizados como promotores de crescimento é necessário que estes se enquadrem dentro de uma escala de características desejáveis, como: não ser tóxico e nem patogênico, apresentar uma exata identificação taxonômica, possuir persistência no trato gastrointestinal, ser capaz de competir com a microbiota residente, apresentar antagonismo em relação a bactérias patogênicas, dentre outras (GAGGÍA et al., 2010).

$O$ perfil de enzimas produzidas por microrganismos também destaca-se entre potentes probióticos (ALMEIDA et al., 2014). Gaggía et al. (2010) descrevem as principais espécies listadas na literatura que apresentam perfil probiótico: Aspergillus oryzae, A. Niger, Saccharomyces cerevisiae, S. pastorianus, Bifidobacterium thermophilum, Lactobacillus acidophilus, L. amylovorus, etc.

Experimentos foram conduzidos a fim de se estudar o efeito da administração de culturas de fungos anaeróbios Orpinomyces KNGF-2 ou suas enzimas sobre a fermentação ruminal, a atividade microbiana no rúmen, e a digestão de nutrientes em geral em ovinos. Observou-se com a administração fúngica na dieta de ovinos gerou uma maior digestibilidade de nutrientes e maior retenção de nitrogênio $(\mathrm{N})$ no rúmen, resultante de um aumento no número de bactérias e fungos e alteração dos padrões de ácidos graxos voláteis (AGVs). No entanto, a administração de enzimas fúngicas não conseguiu melhorar a digestibilidade dos nutrientes, a retenção de $\mathrm{N}$, e os parâmetros da fermentação ruminal. Os resultados indicam que essas proteínas possam ser rapidamente degradadas pela microbiota do rúmen (LEE et al., 2000).

Lee et al. (2004) avaliaram o efeito da suplementação de culturas do fungo anaeróbico rúmen, Piromyces communis (AFC), do AFC filtrado e do AFC autoclavado quanto à sua capacidade de influenciar a produção cumulativa de gás, a digestão da celulose e a população microbiana. A adição de AFC, AFC filtrado e AFC autoclavado 
provocou um aumento acentuado na produção de gás (50, 29 e 32\%, respectivamente). A adição de AFC também aumentou não só o número de bactérias totais e fungos anaeróbios celulolíticos, mas também as atividades enzimáticas de avicelase, carboximetil celulase (CMCase) e xilanase em comparação com o tratamento controle.

Kala et al. (2017) avaliaram dietas isonitrogenadas, variando níveis de fibra detergente neutro (NDT) (70, 85 e 100\%) e observaram que a dieta com maior nível de NDT foi a que apresentou a maior diversidade de enzimas no rúmen de búfalos, principalmente das ativas na degradação de fibras e amido.

Em outro estudo, pesquisadores na área de ruminantes desenvolveram métodos para prétratar a biomassa lignocelulósica da fibra e verificar de que forma ocorre a modulação da população bacteriana do rúmen, bem como a atividade enzimática e metanogênica deste ambiente. Os autores observaram que a biomassa tratada produziu 1,5 $(x)$ vezes mais metano, quando comparada a não tratada. Foi demonstrado também uma variação na composição microbiana e no perfil de bactérias celulolíticas e xilanolíticas (BABA et al., 2017).

Enzimas exógenas de fungos e bactérias já tem sido suplementadas e estudadas na dieta de ruminantes visando otimizar parâmetros zootécnicos de animais criados a pasto. Ganhos significatvos com a suplementação enzimática tem sido observados, principalmente em dietas de baixa digestibilidade (CAMPESTRINI et al., 2005; REIS et al., 2015).

Esses trabalhos respaldam como é importante o papel das enzimas microbianas sobre o metabolismo ruminal, bem como, sobre a otimização da utilização do alimento pelos animais ruminantes. Contudo, a complexidade do ecossitema microbiano dificulta a elucidação das vias de produção de enzimas no rúmen. Essa é uma temática que necessita de muitos estudos, e com o avanço das pesquisas científicas será possível, quem sabe num futuro próximo, a modulação da mirobiota ruminal para eficiência máxima de suas enzimas.

\section{CONCLUSÃO}

Faltam dados conclusivos a respeito da atividade enzimática de diferentes microrganismos, principalmente quando se trata do potencial probiótico visando esta via de ação.

Mais estudos deverão ser feitos a fim de elucidar vias metabólicas, produção de enzimas, função e relevância de diversos microrganismos no rúmen, bem como suas aplicações no ramo biotecnológico.

As enzimas microbianas são essenciais ao perfeito funcionamento do rúmen em animais de produção. Futuros estudos devem ser feitos buscando elucidar as vias modulatórias para eficiência máxima de enzimas nesses ambientes.

\section{AGRADECIMENTOS}

Ao Instituto Federal Goiano e a Universidade Federal de Minas Gerais (UFMG).

\section{REFERÊNCIAS}

ABEDIN, R. M. A.; HANAFY, A. A. E. ; EL-LATIF, S. A. ; EL-ASSAR S. A.; FADEL M. SH. Ligninolytic Oxidative System of Fungal Egyptian Isolates and their Applications in the Decolorization of Industrial Dyes, Biotechnology \& Biotechnological Equipment, v. 27, n. 6, p. 4269-4275, 2013. https://doi.org/10.5504/BBEQ.2013.0067.

ALMEIDA, P. N. M.; FREITAS, C. E. S.; ABRÃO, F. O.; RIBEIRO, I. C. O.; VIEIRA, E. A.; GERASEEV, L. C.; DUARTE, E. R. Atividade celulolítica de fungos aerobios isolados do rúmen de bovinos leiteiros alimentados com forragens tropicais. Revista Caatinga, Mossoró, v. 27, n. 4, p. 202 - 207, 2014. 
AMANI, M. D.; EL AHWANY; YOUSSEF, A. S. Xylanase production by Bacillus pumilus: Optimization by Statistical and Immobilization Methods. Journal of Agriculture and Biological Sciences. v. 6, n. 3, p. 727-732. 2007.

BABA, A.; BANSAL, M.; KUMAR, S.; BISCHOFF K. M.; SANI, R. K. Improved lignocellulose conversion to biofuels with thermophilic bacteria and thermostable enzymes. Bioresource Technology, v. 128, p. 751-759, 2013. https://doi.org/10.1016/i.biortech.2012.10.145

BABA, Y.; MATSUKI M.; MORI, Y.; SUYAMA, Y.; TADA, C.; FUKUDA, Y.; SAITO, M.; NAKAI, Y. Pretreatment of lignocellulosic biomass by cattle rumen fluid for methane production: Bacterial flora and enzyme activity analysis. Journal of Bioscience and Bioengineering, v. xx, n. xx, p. 1-8, 2016.

BADHAN, A. K.; CHADHA, B. S.; JATINDER, K.; SAINI, H. S.; BHAT, M. K. Production of multiple xylanolytic and cellulolytic enzymes by thermophilic fungus Myceliophthora sp. IMI 387099. Bioresource Technology, v. 98, p. 504-510, 2007. https://doi.org/10.1016/i.biortech.2006.02.009

BEG, Q. K.; KAPOOR, M.; MAHAJAN, L.; HOONDAL, G. S. Microbial xylanases and their industrial applications: a review. Applied Microbiol Biotechnology, v. 56, p. 326-338, 2001. https://doi.org/10.1007/s002530100704

BREWER, D.; TAYLOR, A. Aspergillus fumigatus and Sporormia minima isolated from the rumen of sheep. Journal of General Microbiology. v. 59, p.137-139, 1969. https://doi.org/10.1099/00221287-59-1-137

CAMPESTRINI, E.; DA SILVA, V. T. M.; APPELT, M. D. Utilização de enzimas na alimentação animal. Revista Eletrônica Nutritime, v. 2, n. 6, p. 259-272, 2005.

COUGHLAN, M. P.; LJUNGDAHL, L. G. Biochemistry and Genetics of Cellulose degradation, Comparitive Biochemistry of Fungal and Bacterial cellulolytic enzyme systems. Academic Press Limited, v. 5, p. 11-24, 1988.

DIJKERMAN, R.; BHANSING, D. C. P.; OP DEN CAMP, H. J. M.; DRIFT, C. V. D.; VOGELS, G. D. Degradation of structural polysaccharides by the plant cell-wall degrading enzyme system from anaerobic fungi: An application study. Enzyme and Microbial Technology, v. 2, p. 130-136, 1997. https://doi.org/10.1016/S0141-0229(96)00251-7

FANG, X.; YANO, S.; INOUE, H.; SAWAYAMA, S. Lactose Enhances Cellulase Production by the Filamentous Fungus Acremonium cellulolyticus. Journal of bioscience and bioengineering, v. 106, n. 2, p. 115-120. 2008. https://doi.org/10.1263/ibb.106.115

FLINT, H. J. Molecular genetics of obligate anaerobes from the rumen. FEMS Microbiology Letters. The Netherlands, v. 121, n. 17, p. 259-267, Jan. 1994.

FONTY, G.; CHAVAROT, M.; LEPETIT, J.; CANISTRO, J.; FAVIER, R. Mechanical resistance of wheat straw after incubation in cultures of ruminal cellulolytic microorganisms. Animal Feed Science and Technology, v. 80, p. 297-307, 1999. https://doi.org/10.1016/S0377-8401(99)00061-9 
GAIKWAD, J. S.; MAHESHWARI, R. Localization and Release of 13-Glucosidase in the Thermophilic and Cellulolytic Fungus, Sporotrichum thermophile. Experimental mycology, v. 18, p. 300-310, 1994. https://doi.org/10.1016/S0147-5975(06)80003-4

GAO, J.; WENG, H.; ZHU, D.; YUAN, M.; GUAN, F.; XI, Y. Production and characterization of cellulolytic enzymes from the thermoacidophilic fungal Aspergillus terreus M11 under solid-state cultivation of corn stover. Bioresource Technology, v. 99, p. 7623-7629, 2008. https://doi.org/10.1016/i.biortech.2008.02.005

GAGGìA, F.; MATTARELLI, P.; BIAVATI, B. Probiotics and prebiotics in animal feeding for safe food production. International Journal of Food Microbiology, v. 141, p. S15-S28, 2010. https://doi.org/10.1016/j.ijfoodmicro.2010.02.031

GUTIÉRREZ-ROJAS, I.; MORENO-SARMIENTO, N.; MONTOYA, D. Mecanismos y regulación de la hidrólisis enzimática de celulosa en hongos filamentosos: casos clásicos y nuevos modelos. Revista Iberoamericana de Micología, v. 32, n. 1, p. 1-12, 2015. http://dx.doi.org/10.1016/i.riam.2013.10.009

HALLIWELL, G.; PHILLIPS, T. M.; HALLIWELL, N. Microcrystalline Forms of Cellulose as Substrates for Strains of Clostridium thermocellum and Cellulase Formation. Process Biochemimy. v. 30, n. 3, p. 243-250. 1995. https://doi.org/10.1016/0032-9592(95)85005-8

KALA A.; KAMRA D. N.; KUMAR A.; AGARWAL N.; CHAUDHARY L. C.; JOSHI C. G. Impact of levels of total digestible nutrients on microbiome, enzyme profile and degradation of feeds in buffalo rumen. PLOS ONE, v. 12, n. 2, p. 1-23, 2017. e0172051. https://doi.org/10.1371/journal.pone.0172051.

KARMAKAR, M.; RAY, R. R. Current trends in research and application of microbial cellulases. Research Journal of Microbiology. v. 6, p. 41-53. 2010. https://doi.org/10.3923/im.2011.41.53

LEE, S. S.; HA, J. K.; CHENG, K. J. Influence of an anaerobic fungal culture administration on in vivo ruminal fermentation and nutrient digestion. Animal Feed Science and Technology, v. 88, p. 201217, 2000. https://doi.org/10.1016/S0377-8401(00)00216-9

LEE, S. S.; CHOI, C. K.; AHN, B. H.; MOON, Y. H.; KIM, C. H.; HA, J. K. In vitro stimulation of rumen microbial fermentation by a rumen anaerobic fungal culture. Animal Feed Science and Technology, v.115, p. 215-226, 2004. https://doi.org/10.1016/i.anifeedsci.2004.03.002

LIN, C.; TRAN, D.; LAI, C.; YET-POLE; WU, C. Response surface optimization for ethanol production from Pennisetum Alopecoider by Klebsiella oxytoca THLC0409. Biomass and bioenergy. p. 8, 2010.

MARUI, J.; NORIYUKI, K.; KATO, M.; KOBAYASHI, T.; TSUKAGOSHI, N. Transcriptional activator, AoXInR, mediates cellulose-inductive expression of the xylanolytic and cellulolytic genes in Aspergillus oryzae. FEBS Letters, v. 528, p. 279-282, 2002. https://doi.org/10.1016/S00145793(02)03328-8

MOSTAFA, F. A.; ABD EL ATY, A. A.; HAMED, E. R.; EID B M.; IBRAHIM, N. A. Enzymatic, kinetic and anti-microbial studies on Aspergillus terreus culture filtrate and Allium cepa seeds extract and their 
potent applications. Biocatalysis and Agricultural Biotechnology, v. 5, p. 116-122, 2016. https://doi.org/10.1016/i.bcab.2016.01.005

NADIM, M.; DESHAWARE, S.; SAIDI, N.; ABD-ELHAKEEM, M. A.; OJAMO, H.; SHAMEKH, S. Extracellular Enzymatic Activity of Tuber maculatum and Tuber aestivum Mycelia. Advances in Microbiology, v. 5, p. 523-530, 2015. http://dx.doi.org/10.4236/aim. 2015.57054

NARASIMHA, G.; SRIDEV, A.; VISWANATH, B.; CHANDRA, M. S.; RAJASEKHAR, R. B. Nutrient effects on production of cellulolytic enzymes by Aspergillus niger. African Journal of Biotechnology. v. 5, p. 472-476. 2006.

POURCHER, A. M.; SUTRA, L.; HEBE, I.; MOGUEDET, M.; BOLLET, C.; SIMONEAU, P.; GARDAN, L. Enumeration and characterization of cellulolytic bacteria from refuse of a landfill. FEMS Microbiology Ecology. v. 34, p. 229-241, 2001. https://doi.org/10.1111/i.15746941.2001.tb00774.x

REDDY, G. V.; RAVINDRA BABU, P.; KOMARAIAH, P.; ROY, K. R. R. M.; KOTHARI, I. L. Utilization of banana waste for the production of lignolytic and cellulolytic enzymes by solid substrate fermentation using two Pleurotus species ( $P$. ostreatus and $P$. sajor-caju). Process Biochemistry, v. 38, p. 1457-1462, 2003. https://doi.org/10.1016/S0032-9592(03)00025-6

REIS, R. A.; LARA, E. C.; RABELO, C. H. S. Enzimas na Nutrição de Ruminantes. Anais... In: X Congresso Nordestino de Produção Animal, p. 1-22, 2015.

SHALLOM, D.; SHOHAM, Y. Microbial hemicellulases. Current Opininion Microbiology, v. 6, p. 219228, 2003. https://doi.org/10.1016/S1369-5274(03)00056-0

SRINIVASAN, K.; MURAKAMI, M.; NAKASHIMADA, Y.; NISHIO, N. Efficient Production of Cellulolytic and Xylanolytic Enzymes by the Rumen Anaerobic Fungus, Neocalhnastix fron talk, in a Repeated Batch Culture. Journal of bioscience and bioengineering, v. 91, n. 2, p. 153-158. 2001. https://doi.org/10.1016/S1389-1723(01)80058-X

SUBRAMANIYAN, S.; PREMA, P. Biotechnology of microbial xylanases: enzymology, molecular biology and application. Critical Reviews Biotechnology, v. 21, n. 1, p. 33-46, 2002. https://doi.org/10.1080/07388550290789450

THURSTON, B.; DAWSON, K. A.; STROBEL, H. J. Cellobiose versus glucose utilization by the ruminal bacterium Ruminococcus albus. Applied and Environmental Microbiology. v. 59, n. 1, p. 263 12637, 1993.

WALTER, S.; SCHREMPF, H. The synthesis of the Streptomyces reticuli cellulase (Avicelase) is regulated by both activation and repression mechanisms. Molecular and General Genetics. v. 251, n. 2, p. 186-195, 1995.

WANG, B.; REZENOM, Y. H.; CHO, K.; TRAN J. L.; LEE, D. G.; RUSSELL, D. H., JILL, J. J.; YOUNG, R.; $\mathrm{CHU}, \mathrm{K}$. Cultivation of lipid-producing bacteria with lignocellulosic biomass: Effects of inhibitory compounds of lignocellulosic hydrolysates. Bioresource technology, v. 161, p.162 -170, 2014. https://doi.org/10.1016/i.biortech.2014.02.133 
WANG, T.; CHEN, H.; LU, M. et al. Functional characterization of cellulases identified from the cow rumen fungus Neocallimastix patriciarum W5 by transcriptomic and secretomic analyses. Biotechnology for Biofuels, v. 4, n. 24, 2011. doi: 10.1186/1754-6834-4-24. https://doi.org/10.1186/1754-6834-4-24

YANKE, L. J.; SELINGER, L. B.; LYNN, J. R.; CHENG, K. J. Comparison of the Influence of Carbon Substrates on the Fibrolytic Activities of Anaerobic Rumen Fungi. Anaerobe, v. 2, p. 373-378, 1996. https://doi.org/10.1006/anae.1996.0047

ZHANG, J.; ZHONG, Y.; ZHAO, X.; WANG, T. Development of the cellulolytic fungus Trichoderma reesei strain with enhanced b-glucosidase and filter paper activity using strong artificial cellobiohydrolase 1 promoter. Bioresource Technology, v. 101, p. 9815-9818, 2010. https://doi.org/10.1016/i.biortech.2010.07.078 